\title{
Proprotein Convertase Subtilisin/Kexin Type 9
}

National Cancer Institute

\section{Source}

National Cancer Institute. Proprotein Convertase Subtilisin/Kexin Type 9. NCI Thesaurus.

Code C122918.

Proprotein convertase subtilisin/kexin type 9 ( $692 \mathrm{aa}, \sim 74 \mathrm{kDa}$ ) is encoded by the human PCSK9 gene. This protein is involved in the degradation of low density lipoprotein receptor family proteins. 